\title{
EFEITO DOS PARÂMETROS DE EXTRUSÃO SOBRE AS PROPRIEDADES FUNCIONAIS DE EXTRUSADOS DA FARINHA DE BATATA-DOCE ${ }^{1}$
}

\author{
Alexandra M. BORBA ${ }^{2}$, Silene B. S. SARMENTO ${ }^{2, *}$, Magali LEONEL ${ }^{3}$
}

\begin{abstract}
RESUMO
Farinha de batata-doce (Ipomoea batatas) foi extrusada em equipamento de rosca simples, mantendo-se fixas as temperaturas na $1^{\text {a }}$ e $2^{\mathrm{a}}$ zonas de extrusão $\left(20^{\circ} \mathrm{C}\right.$ e $60^{\circ} \mathrm{C}$, respectivamente). O efeito das variáveis umidade da farinha ( 15,18 e $\left.21 \%\right)$, temperatura na $3^{\mathrm{a}}$ zona $\left(100,120\right.$ e $\left.140^{\circ} \mathrm{C}\right)$ e rotação da rosca $(180,210$ e $240 \mathrm{rpm})$ sobre as características dos extrusados foi investigado utilizando-se metodologia de superfície de resposta. O teor de umidade e a temperatura de extrusão influenciaram significativamente a expansão dos extrusados. O índice de expansão apresentou valores crescentes com a temperatura sob baixos teores de umidade. A dureza dos extrusados e também o índice de absorção de água e o índice de solubilidade em água das farinhas extrusadas não mostraram modelo de regressão significativo com as condições de processo. Quanto à cor das farinhas, o componente L* (luminosidade) apresentou valores crescentes e o parâmetro a*, valores decrescentes com a elevação do teor de umidade até $20-21 \%$. O parâmetro b* e a diferença de cor entre farinhas extrusadas e não extrusadas mostraram valores decrescentes com o aumento da umidade. Palavras-chave: batata-doce, extrusados, índice de expansão, índice de absorção de água, índice de solubilidade em água.
\end{abstract}

\section{SUMMARY}

EFFECT OF EXTRUSION PARAMETERS ON SWEET POTATO EXTRUDATES. Sweet potato flour was processed using a single-screw extruder, with extrusion temperatures in zones 1 and 2 of $20^{\circ} \mathrm{C}$ and $60^{\circ} \mathrm{C}$, respectively. The effect of flour moisture content ( 15,18 and $21 \%)$, barrel temperature in zone $3\left(100,120\right.$ and $\left.140^{\circ} \mathrm{C}\right)$ and screw speed $(180,210$ and 240 rpm) on extrudate attributes was investigated using response surface methodology. Moisture content and extrusion temperature had a significant ( $p<0.05$ ) influence on extrudates expansion. The expansion ratio of the extrudates showed increasing values with temperature under conditions of low moisture content. The firmness of extrudates and also the water absorption index and water solubility index of the pre-gelatinized flours did not show significant regression models with the processing conditions. The flours color was influenced by the moisture content. The component $\mathrm{L}^{*}$ showed increasing values and the parameter a* exhibited decreasing values with the increase of moisture content till $20-21 \%$. The parameter $b^{*}$ and the difference of color between extrudated and non extrudated flours showed increasing values as the moisture decreased.

Keywords: sweet potato, extrudates, expansion index, water absorption index, water solubility index.

\section{1 - INTRODUÇÃO}

Um dos processos industriais que tem se mostrado eficiente na obtenção de produtos alimentícios é a extrusão. Este processo possibilita a obtenção de uma variedade de produtos como os snacks, as farinhas prégelatinizadas e os cereais pré-cozidos. A extrusão é viável no processamento de matérias-primas amiláceas, como é o caso da batata-doce.

O princípio básico da extrusão é converter um material sólido em fluido pela aplicação de calor e trabalho mecânico e forçar sua passagem através de uma matriz para formar um produto com características físicas e geométricas prédeterminadas. O processo promove a gelatinização do amido, a desnaturação e a re-orientação das proteínas, a inativação enzimática, a destruição de algumas substâncias tóxicas e a diminuição da contagem microbiana [29].

\footnotetext{
${ }^{1}$ Recebido para publicação em 08/07/2005. Aceito para publicação em 14/09/2005 (001568).

${ }^{2}$ Departamento de Agroindústria, Alimentos e Nutrição, ESALQ, USP - Caixa Postal 9, CEP: 13418-900, Piracicaba - SP. E-mail: sbssarme@ esalq.usp.br.

${ }^{3}$ Centro de Raízes e Amidos Tropicais, UNESP - Caixa Postal 237, CEP: 18603-970, Botucatu - SP.

*A quem a correspondência deve ser enviada.
}

Uma propriedade dos produtos extrusados é a expansão. A alta pressão existente próxima à descarga da matriz, que consiste de orifícios de diversos formatos, é reduzida quando o produto sai do extrusor, ocasionando a evaporação instantânea da água e a expansão do produto [21, 16, 12]. A rápida evaporação da umidade do produto resulta em um resfriamento adiabático, ocorrendo sua solidificação ou endurecimento [21]. O produto extrusado final passa a ter uma textura que é de grande importância para sua qualidade, pois afeta diretamente a aceitabilidade pelos consumidores e as vendas. O que se deseja em snacks com boas características é que os valores de fraturabilidade e dureza sejam baixos [1].

Durante a extrusão-cocção, a estrutura cristalina organizada dos grânulos de amido é destruída de modo parcial ou total, dependendo da proporção amilose-amilopectina e das variáveis de extrusão [12]. As principais propriedades funcionais do amido extrusado quando disperso em água são a absorção e a solubilidade. Assim, este absorve o líquido rapidamente, formando uma pasta à temperatura ambiente, sem qualquer aquecimento [29]. O aumento da solubilidade com a gelatinização é a base para a produção de alimentos amiláceos instantâneos. 
O índice de solubilidade em água está relacionado à quantidade de sólidos solúveis em uma amostra seca, permitindo verificar o grau de severidade do tratamento, em função da degradação, gelatinização, dextrinização e conseqüente solubilização do amido [10].

A formação de compostos de cor durante o processo de extrusão proporciona importante informação a respeito do grau do tratamento térmico [29], sendo ocasionada por reações de caramelização e de Maillard [20].

Embora o processo de extrusão seja basicamente uma simples operação tecnológica, seu controle é complexo, devido às inúmeras variáveis envolvidas e o desconhecimento da influência da maioria delas sobre o processo. Outro fator a se considerar é a complexa natureza do sistema alimentar, no qual várias mudanças químicas e físicas ocorrem simultaneamente durante o processo de extrusão [14].

As culturas amiláceas tropicais e subtropicais de baixo custo de produção podem ser valorizadas pelo uso da extrusão, com o desenvolvimento de produtos alimentícios diferenciados. Assim, o objetivo do presente trabalho foi o de avaliar o efeito de algumas condições de processamento nas características físicas, físico-químicas e funcionais de extrusados de batata-doce.

\section{2 - MATERIAL E MÉTODOS}

\section{1 - Material}

Para a produção de farinha de batata-doce, foram utilizadas raízes frescas de polpa branca, adquiridas do produtor. As enzimas empregadas nas metodologias analíticas foram a amiloglucosidase de Rhizopus mold, da Sigma (A-7255), a alfa-amilase termoestável (Termamyl 120L), da Novozymes, a pancreatina, da Sigma (P-3292) e a pepsina, da Sigma (P-7000).

\section{2 - Métodos}

\subsection{1 - Obtenção da farinha de batata-doce}

As raízes foram descascadas manualmente e imersas em solução de bissulfito de sódio (0,5\%), enquanto aguardavam o corte (chips) em triturador. O material cortado também foi imerso em solução de bissulfito de sódio $(0,5 \%)$ durante 10 minutos para branqueamento. A desidratação $\left(40^{\circ} \mathrm{C}\right)$ foi efetuada em estufa com circulação e renovação de ar por 12 horas. O material desidratado foi triturado em moinho de facas e acondicionado em embalagens plásticas (polietileno) para posterior extrusão.

\subsection{2 - Condições de extrusão}

O processamento foi realizado em linha completa de extrusão da Imbramaq (Ribeirão Preto, Brasil), modelo IMBRA RX, com motor de $10 \mathrm{HP}$ acoplado a redutor de velocidade, sistema de extrusão por fricção mecânica, rosca simples, sistema de refrigeração hidráulica para controle de temperatura na camisa de extrusão, velocidade variável e capacidade de produção de $45 \mathrm{~kg} \cdot \mathrm{h}^{-1}$. Os parâmetros do extrusor mantidos fixos foram: temperatura de extrusão na $1^{\mathrm{a}}\left(20^{\circ} \mathrm{C}\right)$ e na $2^{\mathrm{a}}$ zona $\left(60^{\circ} \mathrm{C}\right)$; largura da rosca ( $14 \mathrm{~mm})$; profundidade da rosca $(4,5 \mathrm{~mm})$; abertura da matriz (5 mm); taxa de compressão da rosca (1:3); taxa de alimentação (200 g.min ${ }^{-1}$ ) e velocidade de corte (125 rpm). Os parâmetros umidade da matéria-prima (15 a $21 \%$ ), temperatura na $3^{\mathrm{a}}$ zona $\left(100\right.$ a $140^{\circ} \mathrm{C}$ ) e rotação de rosca ( 180 a $240 \mathrm{rpm}$ ) variaram conforme o estabelecido no delineamento experimental.

Os produtos obtidos na forma de extrusados foram primeiramente medidos, para cálculo do índice de expansão e, após desidratação em estufa a $45^{\circ} \mathrm{C}$ por 12 horas, foram avaliados quanto à textura (dureza). A farinha prégelatinizada foi obtida pela trituração dos extrusados em moinho de facas, passada em peneira de malha 60 mesh $(0,250 \mathrm{~mm})$ e armazenada em recipientes de vidro para análises posteriores.

\subsection{3 - Avaliações físicas, físico-químicas e funcionais das farinhas}

A farinha de batata-doce não extrusada foi caracterizada quanto à composição físico-química. Os teores de umidade, proteína bruta, lipídeos e cinzas foram determinados segundo a metodologia da AOAC [5], fibras solúveis e insolúveis segundo o método de ASP et al. [4], o amido de acordo com RICKARD \& BEHN [27], os açúcares segundo SOMOGY \& NELSON [30], a amilose de acordo com a ISO 6647 [24] e o pH segundo as normas analíticas do INSTITUTO ADOLFO LUTZ [23].

As farinhas de batata-doce antes e após extrusão foram fixadas sobre suportes (stubs), utilizando-se fita adesiva dupla face. Após fixação, a farinha foi coberta com uma fina camada de ouro em metalizador (Balzers). O aspecto geral das farinhas foi avaliado em microscópio eletrônico de varredura (Zeiss), modelo DSM 940A.

O índice de expansão dos extrusados foi calculado pela relação entre o diâmetro da amostra e o diâmetro da matriz, conforme a metodologia proposta por FAUBION \& HOSENEY [15]. Foram tomadas as medidas de 10 diferentes extrusados dentro de cada tratamento.

A dureza dos extrusados foi determinada em texturômetro Texture Test System, série 2053, modelo TP1, acoplado a um registrador automático de variação de força. O instrumento operou com sensor eletrônico de 300 libras-força (lbf) e a velocidade de descida do cabeçote foi de $30 \mathrm{~cm} \cdot \mathrm{min}^{-1}$. As amostras foram posicionadas horizontalmente na plataforma e uma faca de aço retangular (Single Blade Shear Cell) foi utilizada para romper a amostra. Foram avaliados 10 extrusados por amostra.

O Índice de Absorção da Água (IAA) e Índice de Solubilidade em Água (ISA) foram determinados nas farinhas segundo metodologia de ANDERSON, CONWAY, GRIFFIN [2]. 
A cor foi avaliada utilizando-se o colorímetro Minolta Chroma Meter CR 200b. Os resultados foram expressos em valores $\mathrm{L}^{*}, \mathrm{a}^{*}$ e b [7], sendo que os valores de $\mathrm{L}^{*}$ (luminosidade ou brilho) variam do preto (0) ao branco (100), os valores de a* variam do verde $(-60)$ ao vermelho $(+60)$ e os valores de $b^{*}$ variam do azul ao amarelo, ou seja, de -60 a +60 , respectivamente. Os resultados também foram apresentados em termos de diferença de cor (DCO) entre farinhas antes e após extrusão. Para tal, foi utilizada a Equação 1:

$$
\begin{aligned}
& \mathrm{DCO}=\left[\triangle \mathrm{L}^{2}+\triangle \mathrm{a}^{2}+\triangle \mathrm{b}^{2}\right]^{1 / 2}, \text { onde: } \triangle \mathrm{L}=\mathrm{L}_{\mathrm{o}}-\mathrm{L}_{\mathrm{e}} ; \\
& \triangle \mathrm{a}=\mathrm{a}_{\mathrm{o}}-\mathrm{a}_{\mathrm{e}} ; \triangle \mathrm{b}=\mathrm{b}_{\mathrm{o}}-\mathrm{b}_{\mathrm{e}}
\end{aligned}
$$

Onde:

$\triangle \mathrm{L}=\mathrm{L}_{\mathrm{o}}$ (luminosidade da farinha não extrusada) $-\mathrm{L}_{\mathrm{e}}$ (luminosidade da farinha extrusada);

$\triangle \mathrm{a}=\mathrm{a}_{\mathrm{o}}$ (valor de $\mathrm{a}^{*}$ da farinha não extrusada) - $\mathrm{a}_{\mathrm{e}}$ (valor de a* da farinha extrusada);

$\triangle \mathrm{b}=\mathrm{b}_{\mathrm{o}}$ (valor de $\mathrm{b}^{*}$ da farinha não extrusada) $-\mathrm{b}_{\mathrm{e}}$ (valor de $\mathrm{b}^{*}$ da farinha extrusada).

\subsection{4 - Delineamento experimental}

Para analisar o efeito combinado das variáveis independentes ou preditoras nas características dos extrusados, optou-se pela metodologia de superfície de resposta do tipo central composto rotacional de $2^{\mathrm{a}}$ ordem [9]. O experimento foi conduzido num delineamento fatorial incompleto $3^{3}$ [8] com três fatores ou variáveis independentes (umidade da farinha, temperatura e rotação da rosca do extrusor) e três níveis codificados.

O processamento dos dados e a análise estatística foram realizados com o auxílio do RSREG do sistema SAS versão 8.2 [28]. A significância do modelo foi testada pela análise de variância (ANOVA). Foi adotado o nível de significância alfa de $10 \%(\alpha=0,10)$.

A expressão geral utilizada para predizer o comportamento de cada resposta avaliada pode ser escrita pela Equação 2:

$\mathrm{Y}_{\mathrm{i}=} \beta_{0}+\beta_{1} \mathrm{X}_{1}+\beta_{2} \mathrm{X}_{2}+\beta_{3} \mathrm{X}_{3}+\beta_{11} \mathrm{X}_{1}{ }^{2}+\beta_{22} \mathrm{X}_{2}{ }^{2}+\beta_{33} \mathrm{X}_{3}{ }^{2}+$ $\beta_{12} X_{1} X_{2}+\beta_{13} X_{1} X_{3}+\beta_{23} X_{2} X_{3}$

Onde:

$\mathrm{Y}_{\mathrm{i}}=$ função resposta;

$\mathrm{X}_{1}, \mathrm{X}_{2}, \mathrm{X}_{3}=$ valores das variáveis independentes;

$\beta_{1}, \beta_{2}, \beta_{3}=$ coeficientes lineares estimados pelo método dos quadrados mínimos;

$\beta_{11}, \beta_{22}, \beta_{33}=$ coeficientes das variáveis quadráticas;

$\beta_{12}, \beta_{13}, \beta_{23}=$ coeficiente de interação entre as variáveis independentes.

O delineamento experimental empregado no processo de extrusão da farinha de batata-doce, incluindo os valores codificados e reais das variáveis independentes, são apresentados na Tabela 1 .
TABELA 1 - Delineamento experimental do processo de extrusão

\begin{tabular}{|c|c|c|c|c|c|c|}
\hline \multirow{2}{*}{ Tratamentos } & \multicolumn{3}{|c|}{$\begin{array}{l}\text { Variáveis } \\
\text { codificadas }\end{array}$} & \multicolumn{3}{|c|}{$\begin{array}{c}\text { Variáveis } \\
\text { independentes }\end{array}$} \\
\hline & $\mathrm{X} 1$ & $\mathrm{X} 2$ & $\mathrm{X} 3$ & $\mathrm{X} 1$ & $\mathrm{x} 2$ & X3 \\
\hline 1 & -1 & -1 & -1 & 15 & 100 & 180 \\
\hline 2 & 1 & -1 & -1 & 21 & 100 & 180 \\
\hline 3 & -1 & 1 & -1 & 15 & 140 & 180 \\
\hline 4 & 1 & 1 & -1 & 21 & 140 & 180 \\
\hline 5 & -1 & -1 & 1 & 15 & 100 & 240 \\
\hline 6 & 1 & -1 & 1 & 21 & 100 & 240 \\
\hline 7 & -1 & 1 & 1 & 15 & 140 & 240 \\
\hline 8 & 1 & 1 & 1 & 21 & 140 & 240 \\
\hline 9 & 0 & 0 & 0 & 18 & 120 & 210 \\
\hline 10 & 0 & 0 & 0 & 18 & 120 & 210 \\
\hline 11 & 0 & 0 & 0 & 18 & 120 & 210 \\
\hline 12 & 0 & 0 & 0 & 18 & 120 & 210 \\
\hline 13 & 0 & 0 & 0 & 18 & 120 & 210 \\
\hline 14 & 0 & 0 & 0 & 18 & 120 & 210 \\
\hline 15 & $-\alpha$ & 0 & 0 & 13 & 120 & 210 \\
\hline 16 & $+\alpha$ & 0 & 0 & 23 & 120 & 210 \\
\hline 17 & 0 & $-\alpha$ & 0 & 18 & 86 & 210 \\
\hline 18 & 0 & $+\alpha$ & 0 & 18 & 154 & 210 \\
\hline 19 & 0 & 0 & $-\alpha$ & 18 & 120 & 160 \\
\hline 20 & 0 & 0 & $+\alpha$ & 18 & 120 & 260 \\
\hline
\end{tabular}
da farinha de batata-doce

Onde: $\mathrm{X} 1=$ umidade das amostras $(\%) ; \mathrm{X} 2=$ temperatura do processo $\left({ }^{\circ} \mathrm{C}\right)$; $\mathrm{X} 3=$ rotação da rosca (rpm)

\section{3 - RESULTADOS E DISCUSSÃo}

\section{1 - Caracterização da matéria-prima}

A farinha de batata-doce não extrusada apresentou pH 5,7. A composição da farinha foi de 8,7\% de umidade, $58,1 \%$ de amido, 10,5\% de amilose, 17,0\% de açúcares solúveis totais, $6,7 \%$ de proteínas, 0,7\% de lipídeos, 2,7\% de cinzas, $4,3 \%$ de fibras insolúveis e $1,7 \%$ de fibras solúveis. A farinha de batata-doce é, portanto, um alimento basicamente amiláceo, com teor considerável de açúcares simples e baixo conteúdo lipídico.

\section{2 - Microscopia eletrônica de varredura}

$\mathrm{O}$ aspecto geral das farinhas de batata-doce antes e após extrusão pode ser avaliado pelas micrografias em microscópio eletrônico de varredura (Figura 1). Como não foram observadas diferenças entre os tratamentos de extrusão quanto ao aspecto geral, apenas as fotos do tratamento 8 são apresentadas (Figura $1_{C}$ e $1_{D}$ ).

Na farinha não extrusada (Figura $1_{\mathrm{A}}$ e $1_{\mathrm{B}}$ ) os componentes apresentaram-se soltos ou aglomerados, porém íntegros. Pelas fotos podem ser identificados grânulos de amido em seus formatos originais e materiais não amiláceos, que se acham aderidos entre si ou aos grânulos de amido. Pela Figura $1_{\mathrm{C}}$ observa-se que a farinha de batata- 

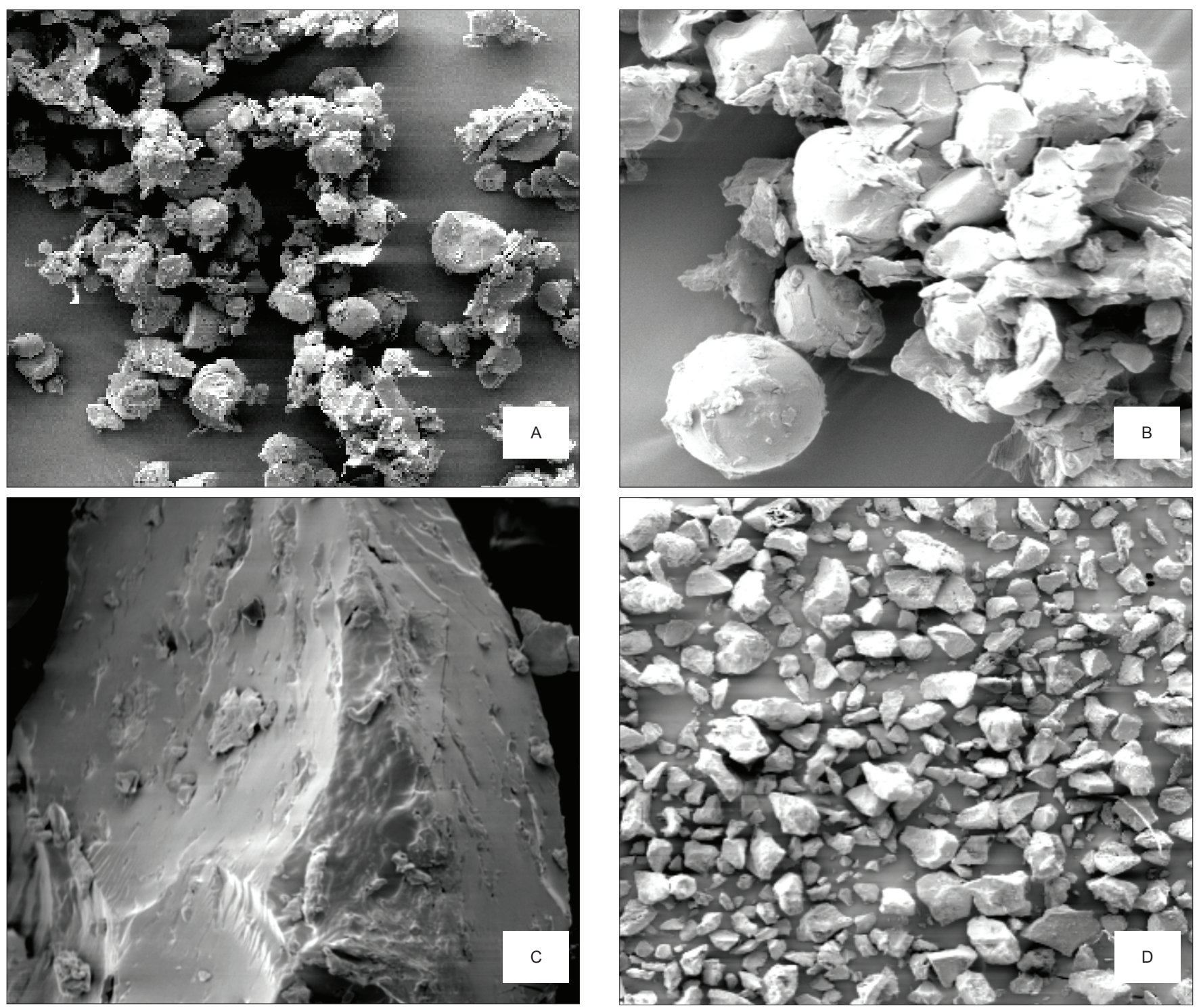

FIGURA 1 - Micrografias das farinhas de batata-doce em microscópio eletrônico de varredura sob diferentes aumentos. Farinha de batatadoce antes da extrusão: A) aumento 20x; B) aumento 100x. Farinha de batata-doce extrusada: C) aumento 20x; D) aumento 500x

doce extrusada apresenta-se como uma massa compacta, amorfa, onde não é possível distinguir grânulos de amido e material não amiláceo. Na Figura $1_{\mathrm{D}}$ observa-se que o material extrusado apresenta superfície irregular, com partes lisas, partes estriadas e alguns orifícios.

\section{3 - Características tecnológicas dos extrusados e das farinhas pré-gelatinizadas}

As propriedades dos extrusados e das farinhas obtidas em função das variáveis independentes testadas podem ser visualizadas na Tabela 2 . Os extrusados foram avaliados quanto ao índice de expansão (IE) e dureza (DUR). As farinhas extrusadas foram avaliadas quanto à cor $\left(\mathrm{L}^{*}, \mathrm{a}^{*}\right.$, $\mathrm{b}^{*}$ ) e diferença de cor (DCO) com relação à farinha não extrusada, índice de absorção de água (IAA) e índice de solubilidade em água (ISA).

\subsection{1 - Índice de expansão dos extrusados}

O IE dos extrusados variou de 1,9 a 2,6 com os diversos tratamentos. O modelo de regressão adotado para o índice de expansão foi significativo $(\mathrm{p}<0,05)$. Dentre os fatores que compõem o modelo, a umidade $(\mathrm{p}=0,02)$ e a temperatura $(\mathrm{p}=0,07)$ afetaram significativamente $\mathrm{o} \mathrm{IE}$, ao passo que não foi observado o efeito significativo $(p>0,10)$ da rotação da rosca. O coeficiente de correlação múltipla $\left(\mathrm{R}^{2}\right)$ foi de $73,6 \%$, indicando um bom ajuste do modelo aos dados, o que garante a validade das predições efetuadas. O modelo total ajustado de $2^{\text {a }}$ ordem para índice de expansão dos extrusados é apresentado na Equação 3:

$-3,93+0,201 \mathrm{U}+0,080 \mathrm{~T}-0,0018 \mathrm{R}+0,0015 \mathrm{U}^{2}-0,000157 \mathrm{~T}^{2}$

$+0,0000312 R^{2}$

$-0,00179 \mathrm{UT}-0,000417 \mathrm{UR}-0,000033 \mathrm{TR}\left(\mathrm{R}^{2}:\right.$ 73,58\%) (3) 
Tabela 2 - Efeito dos tratamentos de extrusão sobre as variáveis dependentes avaliadas nos extrusados e nas farinhas extrusadas

\begin{tabular}{|c|c|c|c|c|c|c|c|c|c|c|c|}
\hline \multirow[t]{2}{*}{ Tratamento } & \multicolumn{3}{|c|}{$\begin{array}{c}\text { Variáveis } \\
\text { independentes }\end{array}$} & \multicolumn{8}{|c|}{ Variáveis dependentes } \\
\hline & $\mathrm{x} 1$ & $\mathrm{X} 2$ & X3 & IE & DUR & IAA & ISA & $L^{*}$ & $a^{*}$ & $\mathbf{b}^{*}$ & DCO \\
\hline 1 & 15 & 100 & 180 & 2,34 & 35,5 & 5,42 & 13,14 & 84,30 & 0,67 & 22,73 & 18,34 \\
\hline 2 & 21 & 100 & 180 & 2,17 & 48,3 & 6,19 & 17,81 & 88,03 & $-1,63$ & 15,07 & 10,15 \\
\hline 3 & 15 & 140 & 180 & 2,52 & 54,0 & 6,16 & 17,34 & 82,80 & 1,83 & 24,63 & 20,89 \\
\hline 4 & 21 & 140 & 180 & 2,15 & 57,5 & 5,96 & 12,57 & 85,47 & $-1,93$ & 18,23 & 14,12 \\
\hline 6 & 21 & 100 & 240 & 2,18 & 42,1 & 5,34 & 12,44 & 84,23 & 1,63 & 19,73 & 16,13 \\
\hline 7 & 15 & 140 & 240 & 2,60 & 48,2 & 5,00 & 14,13 & 85,03 & $-0,20$ & 21,27 & 16,73 \\
\hline 8 & 21 & 140 & 240 & 1,85 & 41,0 & 5,44 & 12,43 & 85,77 & $-1,53$ & 17,50 & 14,07 \\
\hline 9 & 18 & 120 & 210 & 2,28 & 51,2 & 5,15 & 13,65 & 86,80 & $-1,37$ & 17,47 & 12,65 \\
\hline 10 & 18 & 120 & 210 & 2,42 & 59,6 & 5,45 & 13,22 & 85,77 & $-1,17$ & 19,73 & 15,06 \\
\hline 14 & 18 & 120 & 210 & 2,37 & 50,2 & 5,48 & 10,67 & 85,43 & $-1,40$ & 17,90 & 13,85 \\
\hline 15 & 13 & 120 & 210 & 2,48 & 52,9 & 5,83 & 13,13 & 78,77 & 4,60 & 28,20 & 26,50 \\
\hline 16 & 23 & 120 & 210 & 2,17 & 43,3 & 5,73 & 17,18 & 86,57 & $-1,83$ & 17,50 & 12,85 \\
\hline 17 & 18 & 86 & 210 & 1,87 & 39,1 & 5,23 & 13,03 & 81,27 & 1,37 & 24,07 & 21,30 \\
\hline 18 & 18 & 154 & 210 & 2,34 & 54,7 & 5,51 & 12,98 & 84,80 & $-0,30$ & 22,20 & 17,32 \\
\hline 19 & 18 & 120 & 160 & 2,31 & 46,4 & 4,98 & 12,36 & 86,63 & $-0,13$ & 20,73 & 15,41 \\
\hline 20 & 18 & 120 & 260 & 2,42 & 53,0 & 5,24 & 14,57 & 83,63 & 0,37 & 21,10 & 17,49 \\
\hline
\end{tabular}

Sendo: $\mathrm{X} 1=$ umidade $(\%) ; \mathrm{X} 2=$ temperatura do extrusor $\left({ }^{\circ} \mathrm{C}\right) ; \mathrm{X} 3=$ rotação da rosca $(\mathrm{rpm}) ; \mathrm{IE}=$ índice de expansão; DUR=dureza $\left(\mathrm{lbf} . \mathrm{g}^{-1}\right) ; \mathrm{L}^{*}=$ luminosidade; $\mathrm{a}^{*}=$ vermelho/verde; $\mathrm{b}^{*}=$ amarelo/azul; DCO=diferença de cor; IAA=índice de absorção de água ( $\mathrm{g}$. $\mathrm{g}^{-1}$ gel); ISA=índice de solubilidade em água (\%)

A Figura 2 mostra o efeito da umidade da mistura e da temperatura do extrusor no índice de expansão quando a rotação do extrusor foi mantida no ponto central (210 rpm). Sob baixos teores de umidade, os extrusados apresentaram aumento de IE com a elevação da temperatura. Os menores IE foram obtidos principalmente com baixas umidades e temperaturas e também sob altas umidades e temperaturas.

SEBIO [29] encontrou comportamento semelhante ao deste trabalho ao extrusar farinha de inhame. Segundo o autor, a umidade e a temperatura tiveram efeitos evidentes na propriedade de expansão do extrusado, que variou de 1,2 a 2,6. Os maiores valores de expansão foram obtidos em temperaturas altas e umidades baixas. Quando em altas umidades e temperaturas, os extrusados de inhame apresentaram menores valores de expansão.

CHANG \& EL-DASH [11] também encontraram resultados semelhantes em extrusados de amido de mandioca, onde a umidade e a temperatura afetaram significativamente a expansão do produto. No geral, sob baixos valores de umidade, o aumento da temperatura resultou em aumento do IE. A combinação alta umidade e alta temperatura resultou em redução dos índices de expansão. Segundo os autores, a expansão está relacionada ao grau de gelatinização do amido. Baixos conteúdos de umidade no material podem restringir o fluxo do mesmo dentro do extrusor, aumentando o cisalhamento e o tempo de residência, o que poderia talvez aumentar o grau de gelatinização.
A expansão de materiais amiláceos é inversamente proporcional à umidade do material a ser extrusado [3]. A água tem efeito inverso sobre a expansão agindo como plastificante para materiais amiláceos, reduzindo sua viscosidade e a dissipação da energia mecânica no extrusor e, assim, o produto fica mais denso e o crescimento de bolhas é comprimido [13]. O aumento da quantidade de água durante a extrusão pode mudar a estrutura molecular da amilopectina do material, reduzindo a viscosidade elástica e assim, a expansão [13, 31].

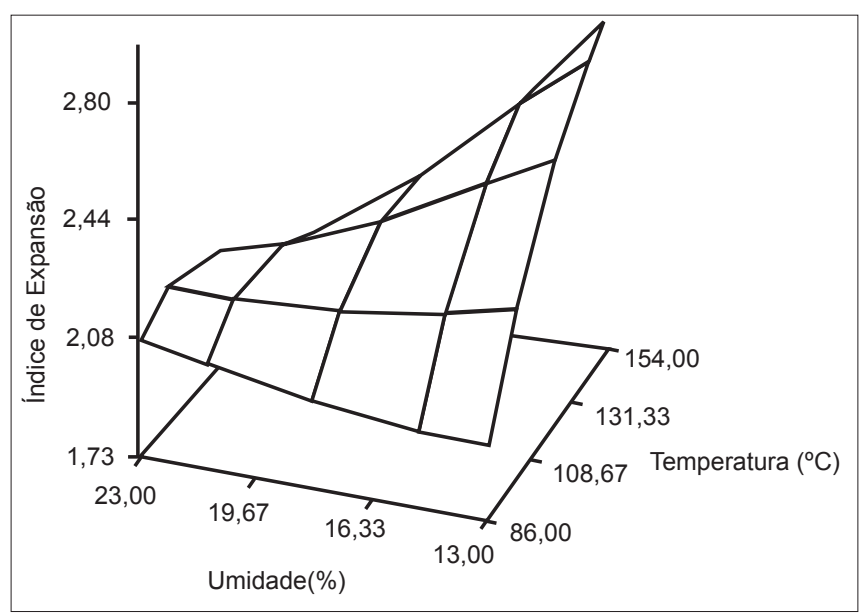

FIGURA 2 - Efeito da variação de umidade das amostras e da temperatura de extrusão sobre o índice de expansão dos extrusados de batata-doce, sob rotação de $210 \mathrm{rpm}$ 


\subsection{2 - Dureza dos extrusados}

A dureza apresentou variação de 35,5 a 59,6 lbf.g ${ }^{-1}$ dentro dos tratamentos. O modelo de regressão adotado para a variação deste parâmetro, entretanto, não foi significativo $(p>0,10)$. O coeficiente de correlação múltipla $\left(\mathrm{R}^{2}\right)$ foi de $58,7 \%$, indicando falta de ajuste do modelo aos dados. O modelo total ajustado de $2^{\mathrm{a}}$ ordem para dureza dos extrusados é apresentado na Equação 4:

$-474,47+18,19 \mathrm{U}+3,13 \mathrm{~T}+1,58 \mathrm{R}-0,21 \mathrm{U}^{2}-0,0055 \mathrm{~T}^{2}$ $-0,0014 R^{2}-0,040 \mathrm{UT}$

$-0,0287 \mathrm{UR}-0,00415 \mathrm{TR}\left(\mathrm{R}^{2}: 58,7 \%\right)$

Os menores valores de dureza foram obtidos a baixas umidades e altas temperaturas para extrusados de farinha de cará [1] e de farinha de inhame [29]. Este último autor atribuiu a menor resistência à ruptura, principalmente à evaporação instantânea da água superaquecida antes da solidificação da estrutura, conferindo ao produto uma característica mais porosa e expandida. Já a maior resistência foi atribuída à baixa pressão interna no extrusor e, conseqüentemente, a uma lenta evaporação da água do produto na saída da matriz, permitindo solidificação da estrutura antes que ocorresse grau adequado de expansão.

\subsection{3 - Índice de absorção de água}

O índice de absorção de água (IAA) das farinhas extrusadas variou de 5,0 a $6,2 \mathrm{~g} \cdot \mathrm{g}^{-1}$ gel, superior ao obtido para farinha não extrusada $\left(3,1 \mathrm{~g} \cdot \mathrm{g}^{-1}\right.$ gel), como esperado.

O modelo de regressão adotado para este parâmetro não foi significativo $(p>0,10)$. O coeficiente de correlação múltipla $\left(R^{2}\right)$ foi de $39,4 \%$, indicando falta de ajuste do modelo aos dados. A Equação 5 representa o modelo total ajustado de $2^{a}$ ordem para índice de absorção de água da farinha extrusada:

$-1,06-0,43 \mathrm{U}+0,0516 \mathrm{~T}+0,072 \mathrm{R}+0,0175 \mathrm{U}^{2}+0,000019 \mathrm{~T}^{2}$ $-0,000093 R^{2}$

- 0,000437UT - 0,000625UR - 0,000223TR (R²: 39,41\%)

Os índices de absorção de água estão relacionados com o grau de degradação das macromoléculas, o que interfere na capacidade das mesmas em absorver água [17, 18]. GUHA, ALI, BHATTACHRYA [18] extrusaram farinha de arroz sob condições de baixa rotação e temperatura e observaram que os valores de IAA apresentaram-se relativamente mais altos que em elevada rotação e temperatura. Segundo os autores, a baixa velocidade do parafuso permite maior tempo de residência da mistura no extrusor e maior cocção das amostras.

\subsection{4 - Índice de solubilidade em água}

O índice de solubilidade em água (ISA) da farinha não extrusada foi de $16,6 \%$ e após a extrusão variou de 10,7 a $22,2 \%$. Estes valores são semelhantes aos encontrados por IWE [25] em misturas de batata-doce e soja extrusadas, ou seja, 15,2 a $19,3 \%$.

Embora tenha havido variação nos valores deste atri- buto nas condições do experimento, o modelo de regressão não foi significativo $(p>0,10)$. O coeficiente de correlação múltipla $\left(\mathrm{R}^{2}\right)$, que foi de $25,4 \%$, indica falta de ajuste do modelo aos dados. O polinômio descrito na Equação 6 corresponde ao modelo total ajustado de $2^{\text {a }}$ ordem para índice de solubilidade em água da farinha extrusada:

$-51,81+2,47 \mathrm{U}+0,62 \mathrm{~T}+0,069 \mathrm{R}+0,0246 \mathrm{U}^{2}-0,00133 \mathrm{~T}^{2}$ $-0,00043 R^{2}$

$-0,026 \mathrm{UT}-0,000375 \mathrm{UR}+0,00084 \mathrm{TR}\left(\mathrm{R}^{2}: 25,4 \%\right)$

A extrusão de farinha de trigo, banana e arroz sob altas temperaturas e baixos teores de água resultou em maior degradação dos grânulos de amido, o que contribuiu para o aumento do ISA [10]. O aumento da velocidade da rosca também resulta em maior fragmentação do amido, devido à maior severidade no tratamento $[22,18]$.

\subsection{5 - Cor das farinhas extrusadas}

O modelo de regressão adotado foi significativo $(p<0,05)$ para o componente $\mathrm{L}^{*}$ (luminosidade). Dentre os fatores que compõem o modelo, a umidade $(p=0,02)$ afetou significativamente o componente $\mathrm{L}^{*}$, ao passo que os efeitos dos fatores temperatura e rotação da rosca de extrusão sobre a luminosidade das farinhas extrusadas não foram significativos. O coeficiente de correlação múltipla $\left(\mathrm{R}^{2}\right)$ foi de $73,4 \%$, indicando bom ajuste do modelo aos dados, o que garante a validade das predições efetuadas. O polinômio descrito na Equação 7 corresponde ao modelo total ajustado de $2^{\text {a }}$ ordem para luminosidade das farinhas instantâneas de batata-doce:

$33,614+5,470 \mathrm{U}+0,146 \mathrm{~T}-0,092 \mathrm{R}-0,090 \mathrm{U}^{2}-0,0017 \mathrm{~T}^{2}$ $+0,000081 R^{2}$

$-0,0016 \mathrm{UT}-0,0072 \mathrm{UR}+0,00143 \mathrm{TR}\left(\mathrm{R}^{2}: 73,44 \%\right)$

O escurecimento conforme a intensidade pode comprometer a cor dos produtos obtidos por extrusão. O componente $L^{*}$ das farinhas extrusadas variou de 81 a 88 , sendo que o valor de $\mathrm{L}^{*}$ na farinha não extrusada foi de 94,6. A extrusão promoveu, portanto, redução da luminosidade das farinhas, isto é, elas se tornaram mais escuras.

A Figura 3 mostra o efeito da umidade das amostras sobre o componente $\mathrm{L}^{*}$ das farinhas extrusadas quando a temperatura e a rotação da rosca foram mantidas no ponto central do delineamento, respectivamente $120^{\circ} \mathrm{C}$ e $210 \mathrm{rpm}$. Foi observado o aumento do componente $\mathrm{L}^{*}$ com o aumento da umidade até $20-21 \%$. A partir deste teor, houve uma redução dos valores de $\mathrm{L}^{*}$.

O modelo de regressão adotado para a variação do componente $a^{*}$ foi significativo $(p<0,03)$ nas condições experimentais. Dentre os fatores que compõem o modelo, a umidade $(p=0,006)$ afetou significativamente o parâmetro $a^{*}$, ao passo que para os fatores temperatura e rotação da rosca não se observou efeito significativo sobre o croma a*. O coeficiente de correlação múltipla $\left(\mathrm{R}^{2}\right)$ foi de $77,3 \%$, indi- 


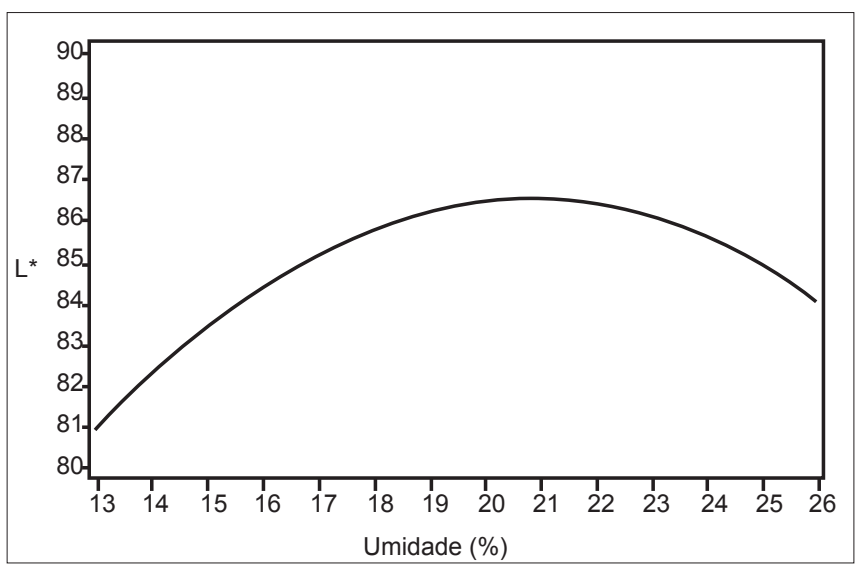

FIGURA 3 - Efeito do teor de umidade da matéria-prima sobre o componente $\mathrm{L}^{*}$ das farinhas extrusadas, sob condições de temperatura de $120^{\circ} \mathrm{C}$ e rotação de $210 \mathrm{rpm}$

cando um ótimo ajuste do modelo aos dados, o que garante a validade das predições efetuadas. O modelo total ajustado de $2^{\text {a }}$ ordem para o parâmetro a* das farinhas extrusadas é apresentado na Equação 8:

$40,752-3,695 \mathrm{U}+0,137 \mathrm{~T}-0,116 \mathrm{R}+0,0707 \mathrm{U}^{2}+$ $0,000811 \mathrm{~T}^{2}+0,000201 \mathrm{R}^{2}$

$-0,00852 \mathrm{UT}+0,00838 \mathrm{UR}-0,00095 \mathrm{TR}\left(\mathrm{R}^{2}: 77,29 \%\right)$

O valor de $\mathrm{a}^{*}$ variou de 4,6 a $-1,9$, o que mostra que houve pequena variabilidade neste parâmetro durante o processo de extrusão, considerando que a variação deste parâmetro vai de $-60 \mathrm{a}+60$. A farinha de batata-doce não extrusada apresentou o valor de $a^{*}$ de $-0,5$, mostrando que o processo aumenta a intensidade deste parâmetro, no caso de alguns tratamentos e diminui a intensidade, no caso de outros. A Figura 4 mostra o efeito da umidade sobre o parâmetro a* quando a temperatura e a rotação da rosca foram mantidas no ponto central $\left(120^{\circ} \mathrm{C}\right.$ e $\left.210 \mathrm{rpm}\right)$. Foi observado que, com o aumento da umidade da matéria-prima (até 21-22\%), o componente a* foi reduzido,

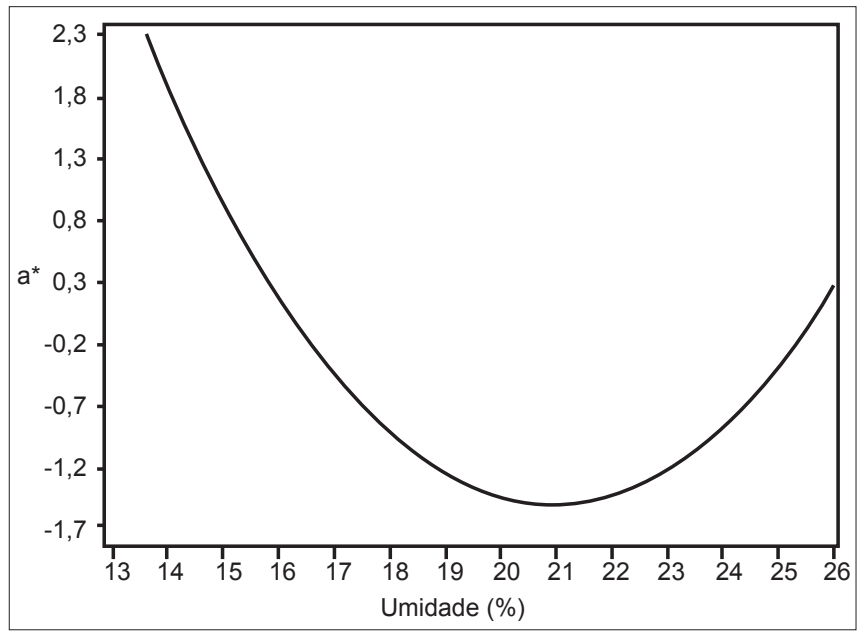

FIGURA 4 - Efeito do teor umidade sobre o parâmetro a* das farinhas instantâneas de batata-doce sob temperatura de $120^{\circ} \mathrm{C}$ e rotação de rosca de $210 \mathrm{rpm}$ passando de $+\mathrm{a}^{*}$ (vermelho) a -a* (verde). Após estes teores (21-22\%), o incremento de umidade nas amostras resultou em aumento da intensidade de $\mathrm{a}^{*}$.

O modelo de regressão adotado foi significativo $(\mathrm{p}<0,003)$ para o valor de $b^{*}$. Dentre os fatores que compóem o modelo, a umidade $(p=0,0007)$ e a temperatura $(p=0,07)$ afetaram significativamente o parâmetro $b^{*}$, ao passo que o efeito do fator rotação da rosca sobre este parâmetro não foi significativo. O coeficiente de correlação múltipla $\left(\mathrm{R}^{2}\right)$ foi de $85,7 \%$, indicando um ótimo ajuste do modelo aos dados, o que garante a validade das predições efetuadas. Na Equação 9 é apresentado o polinômio para modelo total ajustado de $2^{\text {a }}$ ordem para o parâmetro b* das farinhas extrusadas:

$$
\begin{aligned}
& 117,87-7,457 \mathrm{U}-0,324 \mathrm{~T}-0,0343 \mathrm{R}+0,119 \mathrm{U}^{2}+ \\
& 0,0029 \mathrm{~T}^{2}+0,00042 \mathrm{R}^{2}
\end{aligned}
$$$$
+0,00506 \mathrm{UT}+0,00743 \mathrm{UR}-0,00223 \mathrm{TR}\left(\mathrm{R}^{2}: 85,69 \%\right)
$$

O valor de $b^{*}$ variou de 17,5 a 24,7 , sendo que na farinha de batata-doce não extrusada o valor de $b^{*}$ foi de 7,5, ou seja, a extrusão causou uma intensificação do croma amarelo. A Figura 5 mostra o efeito da umidade e temperatura no parâmetro b* quando a rotação da rosca foi mantida no ponto central $(210 \mathrm{rpm})$. Foi observado que, com a redução da umidade, independentemente da temperatura, houve aumento do componente $\mathrm{b}^{*}$, ou seja, aumentou a intensidade do amarelo. Os menores valores do componente $b^{*}$ aconteceram sob elevados valores de umidade e temperaturas intermediárias.

IWE \& NGODDY [26] também verificaram o aumento da cor amarela $\left(+b^{*}\right)$ em farinhas de batata-doce e soja extrusadas sob baixas umidades.

O modelo de regressão adotado para a diferença de cor (DCO) entre as farinhas extrusadas e não extrusadas foi significativo $(p<0,006)$. Dentre os fatores que compõem o modelo, a umidade $(p=0,002)$ e a temperatura $(p=0,10)$ afetaram significativamente a diferença de cor, ao passo

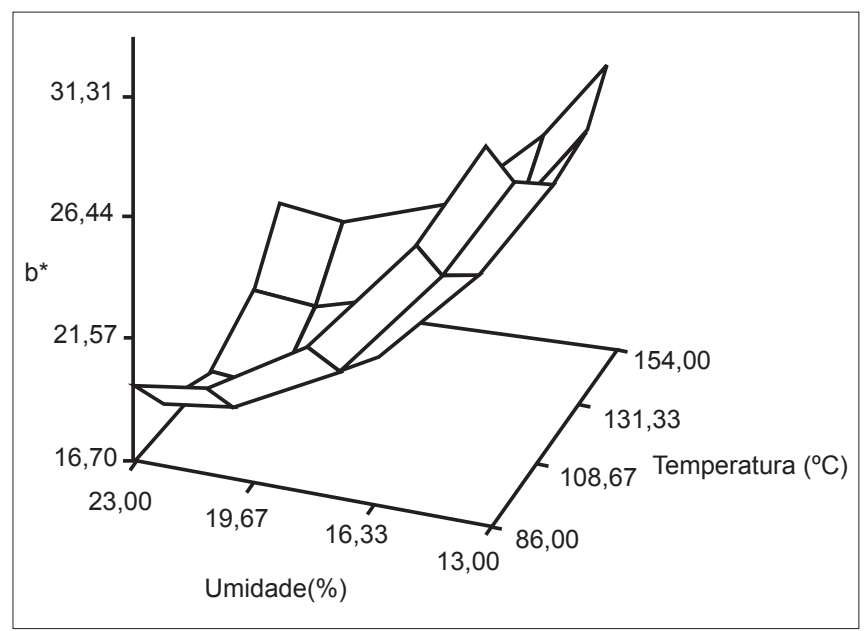

FIGURA 5 - Efeito do teor de umidade da matéria-prima e a temperatura do extrusor no parâmetro b* das farinhas extrusadas, sob rotação de 210 rpm 
que a rotação da rosca não afetou significativamente este parâmetro. O coeficiente de correlação múltipla $\left(\mathrm{R}^{2}\right)$ foi de $83,7 \%$, indicando um ótimo ajuste do modelo aos dados, o que garante a validade das predições efetuadas. A Equação 10 representa o modelo total ajustado de $2^{\mathrm{a}}$ ordem para diferença de cor das farinhas:

$$
\begin{aligned}
& 137,67-9,997 \mathrm{U}-0,371 \mathrm{~T}-0,018 \mathrm{R}+0,161 \mathrm{U}^{2}+0,00321 \mathrm{~T}^{2} \\
& +0,000321 \mathrm{R}^{2} \\
& +0,00604 \mathrm{UT}+0,0113 \mathrm{UR}-0,00252 \mathrm{TR}\left(\mathrm{R}^{2}: 83,71 \%\right)
\end{aligned}
$$

A DCO das amostras extrusadas em relação à amostra natural variou de 10,2 a 26,5. A Figura 6 mostra o efeito da umidade e da temperatura sobre diferença de cor das farinhas extrusadas quando a rotação foi mantida no ponto central (210 rpm). Com a redução da umidade, independentemente da temperatura, a DCO aumentou. Os valores mais baixos de DCO ocorreram em elevada umidade e temperaturas intermediárias.

SEBIO [29], ao extrusar farinha de inhame, verificou que a diferença de cor cresce à medida que a temperatura de processo se eleva, independentemente da variação da rotação da rosca. Os menores valores de DCO ocorreram quando a temperatura e a rotação estavam em seus níveis mais baixos, pois esses níveis não favorecem a ocorrência de reações de escurecimento não enzimático.

O incremento da umidade da farinha no presente estudo até aproximadamente $20-21 \%$ resultou em aumento dos valores L* e redução do croma $+\mathrm{a}^{*}$ para $-\mathrm{a}^{*}$. Já o componente $b^{*}$ e a DCO entre farinha extrusada e não extrusada aumentaram com a redução da umidade. Neste caso, o baixo conteúdo de umidade durante o processamento da farinha de batata-doce favoreceu a ocorrência de reações de escurecimento não enzimático. Segundo BADRIE \& MELLOWES [6], a elevação da temperatura aumenta a intensidade da cor e altos níveis de umidade resultam em produtos mais claros, pois o aumento da umidade reduz o tempo de residência,

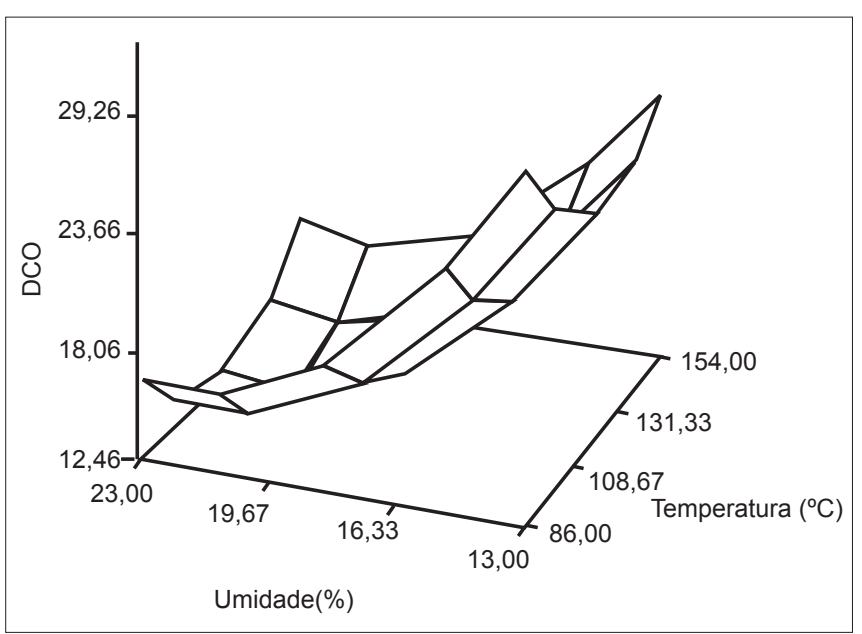

FIGURA 6 - Efeito dos teores de umidade das amostras e temperatura de extrusão na diferença de cor das farinhas de batata-doce, sob rotação de $210 \mathrm{rpm}$ proporcionando menor escurecimento não enzimático dos produtos extrusados.

A cor adquirida pelos produtos extrusados pode ser conseqüência da caramelização ou da reação de Maillard [19], principalmente em materiais que apresentam teores relativamente altos de açúcares totais, como é o caso da batata-doce. A lisina e outros aminoácidos presentes provavelmente reagem com os açúcares redutores, favorecidos pelas condições de processamento, ocorrendo escurecimento dos produtos extrusados.

\section{4 - CONCLUSÕES}

Dentre os parâmetros de processo avaliados (umidade, temperatura e rotação da rosca), a umidade da mistura foi o que exerceu efeito mais pronunciado sobre as características dos produtos obtidos, seguido da temperatura de extrusão.

O índice de expansão dos extrusados mostrou variação significativa com a umidade da mistura e temperatura de processo. Os maiores valores de expansão ocorreram sob condições de baixa umidade e elevada temperatura. Entretanto, sob condições de baixas umidades, a diferença de cor entre produto extrusado e não extrusado é incrementada, ou seja, os extrusados tornam-se mais escurecidos.

O modelo de regressão adotado para a dureza dos extrusados e para o índice de absorção de água e índice de solubilidade em água das farinhas pré-gelatinizadas não foi significativo para os parâmetros de extrusão estudados.

\section{5 - REFERÊNCIAS BIBLIOGRÁFICAS}

[1] ALVES, R.M.L.; GROSSMANN, M.V.E. Parâmetros de extrusão para produção de "snacks" de farinha de cará (Dioscorea alata). Ciência e Tecnologia de Alimentos, v. 22, n. 1, p. 32-38, 2002.

[2] ANDERSON R.A.; CONWAY, V.F.P.; GRIFFIN, E.L. Gelatinization of corn grits by roll- and extrusion-cooking. Cereal Science Today, v. 14, n. 1, p. 4-7, 1969.

[3] ARÊAS, J.A.G. Interações moleculares do amido durante o processo de extrusão. Boletim sbCTA, v. 30, n. 1, p. 28-30, 1996.

[4] ASP, N.G.; JOHANSSON, C.G.; HALLMER, H.; SILJESTRÖM, M. Enzymatic assay of insoluble and soluble dietary fiber. Journal of Agriculture and Food Chemistry, v. 31, n. 3, p. 476-482, 1983.

[5] ASSOCIATION OF OFFICIAL ANALYTICAL CHEMISTS (AOAC). Official Methods of Analysis of the Association of Official Analytical Chemists. $16^{\text {th }} \mathrm{ed}$. Arlington, 1995. $2 \mathrm{v}$.

[6] BADRIE, N.; MELLOWES, W.A. Effect of extrusion variables on cassava extrudates. Journal Food Science, v. 56, p. 1334-1337, 1991.

[7] BIBLE, B.B.; SINGHA, S. Canopy position influences CIELAB coordinates of peach color. HortScience, v. 28, n. 10, p. 992-993, 1993.

[8] BOX, G.E.P.; BEHEKEN, D.W. Some new three level designs for the study of quantitative variables. Technometrics, v. 2, n. 4, p. 455-475, 1960. 
[9] BOX, G.E.P.; HUNTER, W.G.; HUNTER, J.S. Statistics for Experimenters: an Introduction to Design, Data Analysis, and Model Building. New York: John Wiley and Sons, 1978.

[10] CARVALHO, R.V.; ASCHERI, J.L.R.; CAL-VIDAL, J. Efeito dos parâmetros de extrusão nas propriedades físicas de extrusados (3G) de misturas de farinhas de trigo, arroz e banana. Ciência e Agrotecnologia, v. 26, n. 5, p. 1006-1018, 2002.

[11] CHANG, Y.K.; EL-DASH, A.A. Effects of acid concentration and extrusion variables on some physical characteristics and energy requirements of cassava starch. Brazilian Journal of Chemical Engineering, v. 20, n. 2, p. 129-137, 2003.

[12] COLONNA, P.; BULEON, A.; MERCIER, C. Physically modified starch. In: GALLIARD, T. Starch: properties and potential. Chischester: John Wiley \& Sons, 1987. cap. 4, p. 79-114.

[13] DING, Q.; AINSWORTH, P; TUCKER, G.; MARSON, H. The effect of extrusion conditions on the physicochemical properties and sensory characteristics of rice-based expanded snacks. Journal of Food Engineering, v. 66, p. 283-289, 2005.

[14] EL-DASH, A.A. Application and control of thermoplastic extrusion of cereals for food and industrial uses. In: POMERANZ, Y.; MUNCH, L. Cereals, a Renewable Resource: theory and practice. St. Paul: AACC, 1982. cap. 10, p. 165-216.

[15] FAUBION, J.M.; HOSENEY, R.C. High temperature and short time. Extrusion-cooking of wheat starch and flour. I-Effect of moisture and flour type on extrudate properties. Cereal Chemistry, v. 59, n. 6, p. 529-533, 1982.

[16] GIBSON, G.R.; WILLIAMS, C.M. Functional Foods: concept to product. Cambridge: Woodhead Publishing, 2000. $374 \mathrm{p}$.

[17] GOMEZ, M.H.; AGUILERA, J.M. Changes in the starch fraction during extrusion-cooking of corn. Journal of Food Science, v. 48, p. 378-381, 1983.

[18] GUHA, M.; ALI, S.Z.; BHATTACHARYA, S. Twin-screw extrusion of rice flour without a die: effect of barrel temperature and screw speed on extrusion and extrudate characteristics. Journal of Food Engineering, v. 32, p. 251-267, 1997.

[19] GUTKOSKI, L.C.; EL-DASH, A.A. Effect of extrusion process variables on physical and chemical properties of extruded oat products. Plant Foods for Human Nutrition, v. 54, p. 315-325, 1999.
[20] GUTKOSKI, L.C. Extrusão de produtos de aveia. In: GUTKOSKI, L.C.; PEDÓ, I. Aveia: composição química, valor nutricional e processamento. São Paulo: Varela, 2000. cap. 7, p. 169-103.

[21] HARPER, J.M. Extrusion processing of food. Food Technology, v. 32, n. 7, p. 67-72, 1978.

[22] HASHIMOTO, J.M.; GROSSMAN, M.V.E. Effects of extrusion conditions on quality of cassava bran/cassava starch extrudates. International Journal of Food Science and Technology, v. 38, n. 5, p. 511-517, 2003.

[23] INSTITUTO ADOLFO LUTZ. Normas Analíticas do Instituto Adolfo Lutz: métodos químicos e físicos para análise de alimentos. $3^{\mathrm{a}}$ ed. São Paulo, 1985. v. 1.

[24] INTERNATIONAL ORGANIZATION FOR STANDARDIZATION. Norme Internationale: riz détermination de la teneur en amylose. Suisse, 1987. 5 p. (ISO 6647)

[25] IWE, M.O. Effects of extrusion cooking on functional properties of mixtures of full - fast soy and sweet potato. Plant Foods for Human Nutrition, v. 53, p. 37-46, 1998.

[26] IWE, M.O.; NGODDY, P.O. Proximate composition and some functional properties of extrusion cooked soybean and sweet potato blends. Plant Foods for Human Nutrition, v. 53, p. 121-132, 1998.

[27] RICKARD, J.E.; BEHN, K.R. Evaluation of acid and enzyme hydrolytic methods for the determination of cassava starch. Journal of Science of Food and Agriculture, v. 41, n. 4, p. 373-379, 1987.

[28] SAS INSTITUTE, Inc. The SAS System release 8.2. SAS Institute Inc., Carry: NC, 1999.

[29] SEBIO, L. Efeito de alguns parâmetros operacionais de extrusão nas propriedades físico-químicas da farinha de inhame (Dioscorea rotundata). Campinas, 1996. 106 p. Dissertação (Mestrado) - Faculdade de Engenharia de Alimentos, Universidade Estadual de Campinas.

[30] SOMOGY, M. Determination of blood sugar. Journal of Biology Chemistry, n. 160, p. 69-73, 1945.

[31] THYMI, S.; KROKIDA, M.K.; PAPPA, A.; MAROULIS, Z.B. Structural properties of extruded corn starch. Journal of Food Engineering, v. 68, p. 519-526, 2005.

\section{6 - AGRADECIMENTOS}

Os autores agradecem à Fundação de Amparo à Pesquisa do Estado de São Paulo (FAPESP) pelo apoio financeiro ao projeto (Processo 03-05135-0). 